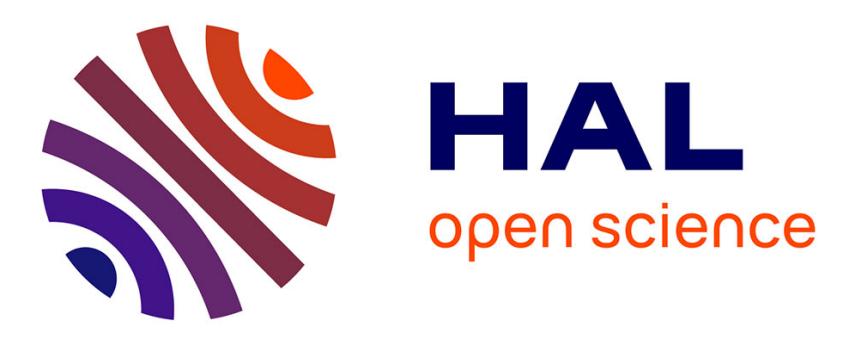

\title{
Evaluation of the Structure-induced Rolling Resistance (SRR) for pavements including viscoelastic material layers
}

\author{
Olivier Chupin, Jean-Michel Piau, Armelle Chabot
}

\section{To cite this version:}

Olivier Chupin, Jean-Michel Piau, Armelle Chabot. Evaluation of the Structure-induced Rolling Resistance (SRR) for pavements including viscoelastic material layers. Materials and structures, 2013 , 46 (4), pp. 683-696, graphiques, schémas, tabl., bibliogr. 10.1617/s11527-012-9925-z . hal-00849470

\author{
HAL Id: hal-00849470 \\ https://hal.science/hal-00849470
}

Submitted on 31 Jul 2013

HAL is a multi-disciplinary open access archive for the deposit and dissemination of scientific research documents, whether they are published or not. The documents may come from teaching and research institutions in France or abroad, or from public or private research centers.
L'archive ouverte pluridisciplinaire HAL, est destinée au dépôt et à la diffusion de documents scientifiques de niveau recherche, publiés ou non, émanant des établissements d'enseignement et de recherche français ou étrangers, des laboratoires publics ou privés. 


\title{
Evaluation of the Structure-induced Rolling Resistance (SRR) for pavements including viscoelastic material layers
}

\author{
O. Chupin ${ }^{\mathrm{a} \bowtie}$, J.-M. Piau ${ }^{\mathrm{a}}$, A. Chabot ${ }^{\mathrm{a}}$ \\ a LUNAM Université, IFSTTAR, IM, Structures, F- 443414 Bouguenais, France. \\ $凶$ corresponding author: Tel. +33 240845 786; fax: +33 240845994 . \\ olivier.chupin@ifsttar.fr
}

\begin{abstract}
This article addresses the question of whether the viscoelastic behavior of asphalt pavements, as part of the factors that impact the rolling resistance, might lead to substantially higher energy consumption of road traffic, as compared to non-dissipative pavements. In the context of sustainable development (fuel consumption, gas emission), this is a current issue for the pavement design community. This problem is tackled by a theoretical approach which consists in computing the Structure-induced Rolling Resistance (SRR) for a vehicle by using the mechanical response of a layered viscoelastic medium excited by moving loads (i.e. tires of the vehicle). The structure-induced power dissipation is shown to be proportional to the integral over contours of the applied loads of the pavement deflection, times the outward normal to these contours. Following the developed approach, the determination of the SRR force engendered by the structure-induced dissipation is obtained from the computation of the deflection of the viscoelastic pavement to an assumed pressure distribution. Such an approach applied to a thick asphalt pavement allows the SRR for a typical road structure to be evaluated as a function of temperature and speed. A non-dimensional analysis is also presented to extend the results to some other contexts. Under the assumptions made for the derivation of the SRR, the main conclusion of the case study is that the extra fuel consumption for a vehicle resulting from the viscoelastic behavior of asphalt pavements is small.
\end{abstract}

Keywords: rolling resistance; moving load; viscoelasticity; layered medium; asphalt pavement

\section{Introduction}

Depending on the traffic volume, the energy use for construction, maintenance and operation of the road is less than 2 to $5 \%$ of the energy used by the traffic itself (estimate obtained from a life cycle assessment and published in EAPA, 2004). Consequently, reducing the energy consumption of the traffic is of overwhelming importance if one wants to mitigate the environmental impact of road pavements. The energy/fuel consumption of the traffic depends on many factors. At the vehicle level, these factors can be listed as follows: thermodynamic efficiency of the engine to transfer heat into mechanical power, driveline losses in the vehicle (EAPA, 2004), rolling resistance (RR), air resistance, inertia resistance (effect of vehicle mass and positive/negative acceleration), and gradient resistance (effect of road slope and vehicle 
weight). As an example, Fig. 1 shows the ratio of these factors to the theoretical energy available in the fuel for a 40-ton truck driving at a constant speed of $80 \mathrm{~km} / \mathrm{h}$ (Sandberg, 2001; EAPA, 2004). Regarding the rolling resistance which is the factor of interest in the present article, it accounts for about $12 \%$ of the total fuel consumption (Fig. 1).
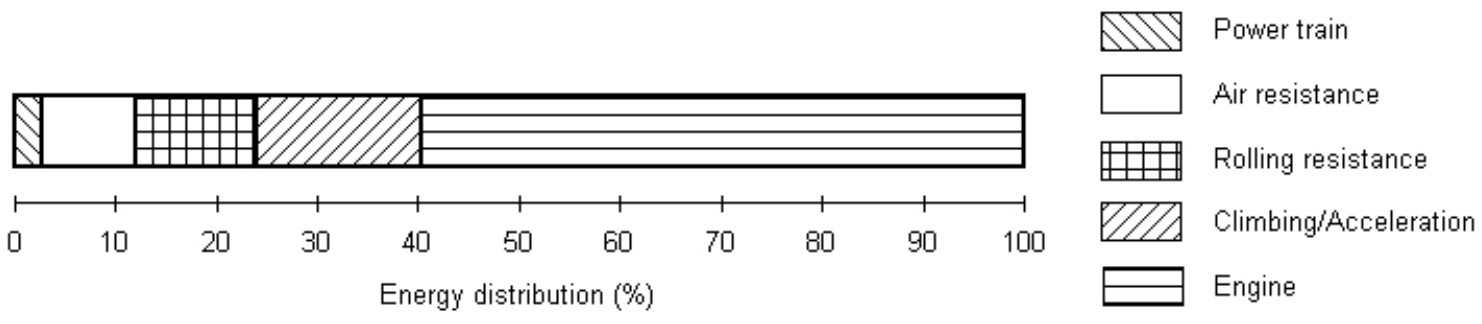

Figure 1. Estimate of the energy distribution of the potential energy available in the fuel, for a truck driving at constant speed on a typical road (after Sandberg, 2001 and EAPA, 2004)

This figure corresponds approximately to $30 \%$ of the available mechanical power output of a heavy truck (the efficiency of the engine being approximately 40\%). Note that the importance of the rolling resistance or air drag is highly dependent on the vehicle speed, but in opposite ways. The rolling resistance indeed decreases with speed on the contrary of air drag. Besides, a study by Glaeser (2005) evaluates the order of magnitude of the rolling resistance to $1 \%$ of the weight of the vehicle (passenger car) which is consistent with the figures above (see Table 1).

Table 1. Power dissipation due to the rolling resistance and obtained from estimates of Figure 1 and a truck of $450 \mathrm{hp}$ rolling at $80 \mathrm{~km} / \mathrm{h}$ (maximum power output)

\begin{tabular}{ll}
\hline Truck engine power & $335 \mathrm{~kW}$ (or 450hp) \\
$\begin{array}{l}\text { Theoretical power of the fuel consumption at maximum truck } \\
\text { power output (efficiency of the engine }=40 \%)\end{array}$ & $837 \mathrm{~kW}(=335 / 40 \%)$ \\
\hline Power dissipation due to Rolling Resistance (RR) & $100 \mathrm{~kW}(=837 * 0.12)$ \\
\hline
\end{tabular}

The rolling resistance itself results from various contributions which are the tire deformation, the pavement surface geometry (slope, texture and evenness) (Zaabar and Chatti, 2010; Bendtsen, 2004; Igwe et al., 2009; Lepert and Brillet, 2009) and the pavement structure. Several works on the evaluation of the rolling resistance for both rigid and asphalt pavements have been carried out (e.g. Zaniewski, 1989; Taylor and Patten, 2006) and tend to confirm that rigid pavements may assist in delivering lower levels of vehicle fuel consumption. However, the results of these research projects are subject to uncertainty since they are derived from experimental studies for which the effect of the SRR is difficult to dissociate from other contributing factors (e.g. texture), especially for experiments performed on road networks. 
Recently, Zaabar and Chatti (2011) attempted to extract the effect of pavement surface texture and type and concluded that, at low speeds and under summer conditions, heavy trucks driven over asphalt pavements consume about $4 \%$ more due to the type of pavement than if they were driven over concrete pavements. Besides, in order to overcome the difficulty of isolating the sole effect of the SRR in measurements performed on road networks, Benbow et al. (2007) have used a pavement testing facility. The conclusions drawn by the authors indicate that the average coefficient of rolling resistance measured on a rigid pavement was about $5 \%$ lower than the one obtained on a flexible pavement but also that the evidence of a difference between the two types of pavement does not support a conclusion that the difference is statically significant. Actually, according to Benbow et al. (2007), it is extremely difficult to reproduce the experiment with exactly the same temperatures and other conditions.

In this paper, the goal is to investigate the RR from the standpoint of the pavement engineer, taking into account the contributing factors which are specific to pavements. These are the pavement surface geometry (slope, evenness), the choice of the surface course and of other pavement layers (structural layers). More specifically, we concentrate on the last aspect which is responsible for what we call the Structure-induced Rolling Resistance (SRR), considering that the three aforementioned factors are widely independent even when it comes to the choice of the surface and the base courses. In particular, we aim at computing the RR due to the viscoelastic behavior of bituminous layers; the underlying practical question being whether the choice of a flexible pavement with an asphalt base course or a rigid pavement with a concrete base course - assumed to be quasi-elastic and non-dissipative if in good shape - has or not a significant impact in terms of traffic fuel consumption, and subsequently whether SRR has to be considered as a pavement design criterion.

To tackle this problem, we use a theoretical approach which makes it possible to eliminate easily all possible sources of dissipation other than the structural layers and to avoid experimental difficulties in doing so.

Note that a similar study about the comparison of the RR induced by different types of surface courses would be of interest as well in the aim of optimizing the whole pavement with regards to the fuel consumption. However such a study is beyond the scope of this paper and would require a theoretical approach different from that presented here further.

The paper is outlined as follows: section 2 presents theoretical considerations for the calculation of SRR and the numerical model used for its computation. Section 3 is devoted to 
the application to a thick asphalt pavement for which quantitative results of SRR as a function of temperature and speed are provided. In section 4, the study is enriched by a non-dimensional analysis that allows heavy trucks and passenger cars to be compared in terms of SRR.

Conclusions are drawn in section 5 .

\section{Theoretical calculation of the SRR}

This section first presents the assumptions of the problem and the theoretical formula utilized to calculate SRR for a vehicle traveling on a layered medium with viscoelastic materials. The rolling resistance force $\left(F_{R R}^{s t r}\right)$ induced by the structural effect is derived from the power dissipation $\left(\wp_{R R}^{s t r}\right)$ which is itself based on the calculation of the deflection at the pavement surface. The numerical procedure that enables the computation of the deflection and the power dissipation is introduced in this section.

\subsection{Assumptions of the problem and derivation of the $\wp_{R R}^{s t r}$ and $F_{R R}^{s t r}$ formulas}

The pavement considered here is a multilayered structure whose layers are assumed to be linear elastic (for soil and non-bituminous materials) or viscoelastic (for asphalt materials). The vehicle tires are supposed to be non-dissipative and a quasi-static regime is assumed. On that account, the vehicle is supposed to move at the constant speed $V$ (no breaking or acceleration effects) and the pavement is viewed as a horizontal semi-infinite medium homogeneous in the driving direction, $x$. According to this assumption, any mechanical field $f(x, y, z, t)$ can be expressed in the load frame as a function $f^{*}(X, y, z)$ with $x=X+V t$. Moreover, the derivatives verify the relation: $\partial f / \partial t=-V \partial f^{*} / \partial X$.

Let us consider now one wheel of the vehicle moving at the constant speed $V$ at the surface of the multilayered structure and a vertical force, $P$, applied to the wheel axle. In order to maintain the quasi-static regime a "driving" force and a possible moment (in the case of a driving wheel) must be applied to the wheel axle (see Fig. 2). This results in a horizontal force, $T_{R R}^{s t r}$, and a moment, $M_{R R}^{s t r}$, that allow balancing the other forces acting on the wheel. These are the vertical force $P$ and the contact forces, $P_{c}$ and $T_{c}$, at the interface between the wheel and the pavement. Note that $P_{c}$ is offset by a distance $e$ from the wheel axis so that the dynamical balance of moment of forces is respected for the wheel (i.e. equal to zero for a constant angular 
velocity). As specified below, $T_{R R}^{s t r}$ and $M_{R R}^{s t r}$ are used to quantify the SRR. Note that for a towed wheel, $M_{R R}^{s t r}$ would be equal to zero.

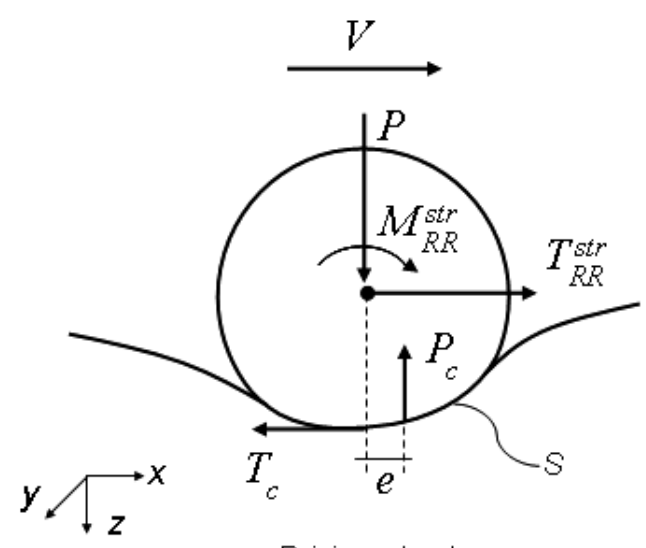

Driving wheel

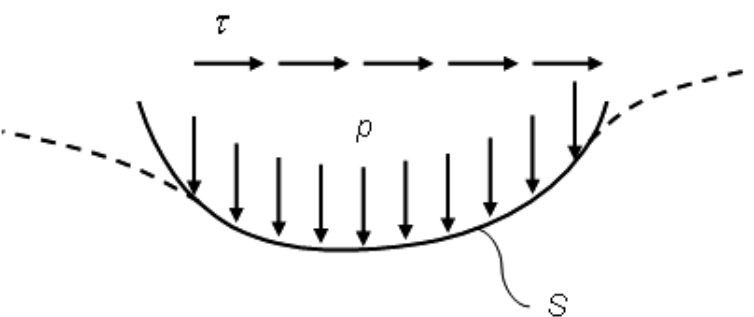

Close-up of the contact surface representation of surface forces

Figure 2. Diagram of resulting forces applied to the wheel

On the basis of the preceding considerations, the power dissipation due to the structural effect can be expressed as follows:

$$
\wp_{R R}^{s t r}=T_{R R}^{s t r} V+M_{R R}^{s t r} \omega=F_{R R}^{s t r} V
$$

in which $\omega$ denotes the angular velocity of the wheel and $F_{R R}^{s t r}$ the global rolling resistance force that we are interested in. Note that according to the convention used here, $F_{R R}^{s t r}$ is positive for a wheel moving in the x-direction. $F_{R R}^{s t r}$ allows to evaluate the rolling resistance in both cases of a towed and a driving wheel. Note that for the assessment of the traffic consumption, the use of $F_{R R}^{s t r}$ is preferable to $\wp_{R R}^{s t r}$. Indeed, $F_{R R}^{s t r}$ also represents the energy consumption per unit length whereas $\wp_{R R}^{s t r}$ must be divided by $V$ to take account of the travel duration.

For the determination of $\wp_{R R}^{s t r}$, Eq.(1) is reformulated using the contact forces at the interface between the wheel and the pavement. To do so, the contact forces are assumed to be associated to uniform distributions of vertical pressure, $p$, and shear stresses, $\tau$, acting on the tire imprint $S$ (Fig. 2). Then:

$$
T_{c}=-T_{R R}^{s t r}=-\tau S, \quad P_{c}=-P=-p S
$$

The choice of uniform pressure distributions is a mathematical choice used to simplify further the computation of $\wp_{R R}^{s t r}$ (resp. $F_{R R}^{s t r}$, see below). Nonetheless, owing to Saint-Venant's 
principle, this simplification with regards to the actual tire/pavement contact pressure is considered to have no significant consequence on the evaluation of the dissipated energy. Indeed, this quantity represents the volume integral over the whole viscoelastic layers of the density of dissipated energy. Therefore, it barely depends on the accurate description of boundary conditions provided that the resultant force is correct (the fact that this quantity can be computed using the local displacements under the tire imprints, as shown below, is purely mathematical and is not in contradiction with this argument). On the other hand, it is supposed that the time variations of the load (e.g. dynamic loading due to evenness) are negligible as compared to our purpose.

Besides, considering the first law of thermodynamics (e.g. Lemaitre and Chaboche, 1996) applied to the wheel yields (with no heat supplied to the system):

$$
\wp_{R R}^{s t r}+\wp_{c}=\frac{d}{d t}\left(K_{\text {wheel }}+E_{\text {wheel }}\right)
$$

where $\wp_{c}$ is the power of contact forces. In Eq.(3), the derivative of the kinetic energy with respect to time ( $d K_{\text {wheel }} / d t$ ) is equal to zero on account of the quasi-static assumption. The rate of internal energy ( $\left.d E_{\text {wheel }} / d t\right)$ is also nil under the assumption of a non-dissipative tire. Finally, the right hand side in Eq.(3) is zero and one has: $\wp_{R R}^{s t r}=-\wp_{c}$.

Another assumption used for the determination of the rolling resistance is that no slip occurs at the tire-pavement contact. The following relation is then verified:

$$
\vec{v}_{\text {wheel }}(Q)=\vec{v}_{\text {pavement }}(Q)
$$

for any point $Q$ that belongs to the contact surface, $S . v_{\text {wheel }}(Q)$ is the velocity vector of point $Q$ that belongs to the wheel. The structure-induced power dissipation due to the rolling of the wheel can now be rewritten as follows:

$$
\wp_{R R}^{s t r}=-\wp_{c}=\int_{S} \tau \frac{d u(x, y, z, t)}{d t} d S+\int_{S} p \frac{d w(x, y, z, t)}{d t} d S
$$

in which $u$ and $w$ are the horizontal ( $x$ direction) and the vertical components of the displacement field at the pavement surface (similar to those of the wheel). Eq.(5) reveals the fact that the structure-induced power dissipation is equal to the power of the contact forces. 
In subsequent calculations, it is assumed that the power of horizontal forces $(\tau)$ can be neglected so that only $p$ needs to be considered in the definition of contact forces. This assumption is consistent with measures performed in a study by Hammoum et al. (2010) that indicates that the magnitude of tangential forces for a vehicle driving at a steady speed are negligible compared to vertical forces. Nevertheless, this assumption will be verified $a$ posteriori in section 3.3. Considering now the quasi-static assumption and using the divergence theorem to transform surface integrals in Eq.(5) into integrals over the contour of the loaded area $(\Gamma)$ yields:

$$
\wp_{R R}^{s t r}=\int_{S} p \frac{d w(x, y, z, t)}{d t} d S=-p V \int_{S} \frac{\partial w^{*}(X, y, z)}{\partial X} d S=-p V \int_{\Gamma} w^{*}(X, y, z) \cdot n_{X} d l
$$

in which $n_{X}$ is the X-component of the outward unit normal to contour $\Gamma$ that encloses the tire imprint. Finally, the rolling resistance force due to the structural effect reads:

$$
F_{R R}^{s t r}=-p \int_{\Gamma} w^{*}(X, y, z) \cdot n_{X} d l
$$

Eq.(7) shows that the calculation of $F_{R R}^{s t r}$ requires the determination of the vertical displacement (or deflection) at the free surface of the pavement subjected to a moving load. Note that in addition to the coordinates $(X, y, z)$ the deflection, $w^{*}$, depends on the pavement structure, the material properties of the layers that compose the pavement, the temperature $(\theta)$ for asphalt materials which are highly thermosensitive, and the speed of the load even though it does not appear explicitly in Eq.(7).

The assumptions and the method used for the computation of $w^{*}(X, y, z)$ are given in sections 2.2 and 2.3 .

In the case of multiple loads moving at the constant speed $V$, Eq.(7) can be extended as follows:

$$
F_{R R}^{s t r}(G)=-\sum_{i=1}^{\text {nloads }} p_{i} \int_{\Gamma_{i}} w^{*}(X, y, z) \cdot n_{X} d l
$$

in which $w^{*}$ is the deflection for the total set of loads. nloads is the total number of loads and $\Gamma_{i}$ represents the contour of the $i t h$ tire imprint. For independent groups of interacting loads, i.e. for groups of loads that, in regards to the deflection, do not interact significantly between 
themselves (e.g. the left and the right side of a wheel axle), $F_{R R}^{s t r}$ can be approximated as follows:

$$
F_{R R}^{\text {str }}(G) \approx \sum_{g=1}^{\text {ngroups }} F_{R R}^{\text {str }}\left(G_{g}\right) \text { with } \quad G_{g}=\bigcup_{i=1}^{\text {nloads }}\left(\Gamma_{g}\right)_{i}
$$

$G_{g}$ denotes the $g$ th independent group of loads and ngroups is the total number of $G_{g}$. $\left(\Gamma_{g}\right)_{i}$ is the ith tire imprint of the independent group $g$. This property is used later in the application to derive the SRR force for a $400 \mathrm{kN}$ truck from the $F_{R R}^{s t r}$ value computed for a semi-axle load of $65 \mathrm{kN}$.

\subsection{Origin of the dissipation and assumptions for the calculation of $w^{*}$}

It is worth giving a physical meaning to Eq.(6) by considering the different sources of dissipation within the pavement structure $\Omega$. To accomplish this, let us consider the principle of virtual power applied to $\Omega$ and to the actual velocity field with the small strain assumption. Since $-\wp_{c}=\wp_{R R}^{s t r}$ is the power of external forces acting on $\Omega$, it yields

$$
\wp_{R R}^{s t r}={\frac{d K^{\text {medium }}}{d t}}+\int_{\Omega} \sigma: \dot{\varepsilon} d \Omega
$$

However, as shown below, the power of internal forces can be replaced by the power dissipation when neglecting the thermo-mechanical effects due to the wheel loads (it can be shown that given the numerical values of the coefficient of thermal expansion of asphalt materials this effect does not play an important role in the problem). Indeed, using the thermodynamics of linear viscoelasticity, the density $\dot{\phi}^{v}$ of dissipated power (viscous dissipation per unit volume) can be written:

$$
\dot{\phi}^{v}(t)=\sigma: \dot{\varepsilon}-\dot{\psi}(t) \text { with } \psi(t)=\frac{1}{2} \int_{-\infty}^{t} \int_{-\infty}^{t} r(2 t-u-v): \dot{\varepsilon}(u): \dot{\varepsilon}(v) d u d v
$$

in which $r$ denotes the relaxation function tensor and $\psi$ the isothermal Helmholtz free energy characterizing the stored elastic energy in the material (Staverman and Schwarzl, 1952a, 1952b). Then, using Eq.(11), the internal virtual power reads:

$$
\int_{\Omega} \sigma: \dot{\varepsilon} d \Omega=\int_{-\infty}^{\infty} d z \int_{-\infty}^{\infty} d y \int_{-\infty}^{\infty}\left(\dot{\phi}^{v}+\dot{\psi}\right) d X
$$


However,

$$
I=\int_{-\infty}^{\infty} \dot{\psi} d X=-V \int_{-\infty}^{\infty} \frac{d \psi^{*}}{d X} d X=-V\left[\psi^{*}(+\infty)-\psi^{*}(-\infty)\right]
$$

with

$$
\psi^{*}(X)=\frac{V^{2}}{2} \int_{-\infty}^{X} \int_{-\infty}^{X} r\left(\frac{2 X-X_{u}-X_{v}}{V}\right): \frac{d \varepsilon^{*}}{d X_{u}}: \frac{d \varepsilon^{*}}{d X_{v}} d X_{u} d X_{v}
$$

So, $\psi^{*}(-\infty)=0$ and $I=-V \psi^{*}(+\infty)$. Because of the condition $\lim _{X \rightarrow \infty} \varepsilon^{*}(X)=0$, it can also be shown that $I=-V \psi^{*}(+\infty)=0$. Consequently, $\int_{\Omega} \sigma: \dot{\varepsilon} d \Omega=\int_{\Omega} \dot{\phi}^{v} d \Omega$ and Eq.(10) becomes:

$$
\wp_{R R}^{s t r}={\frac{d K^{\text {medium }}}{d t}}^{\text {t }}+\int_{\Omega} \dot{\phi}^{v} d \Omega
$$

Eq.(15) shows that the SRR results from two terms: (i) the production of kinetic energy in $\Omega$ that takes the form of mechanical waves propagating towards infinity and dissipating (geometrical and material dissipation), (ii) the viscous dissipation within the bituminous layers of the pavement. The calculation of the deflection, $w^{*}(X, y, z)$, in Eq.(6) must integrate inertia forces to be strictly equivalent to Eq.(15). However, the numerical example of section 3 will show that the contribution of this term is negligible as compared to that of the viscous dissipation.

As expected, in the case of a purely elastic multilayer structure the second term is nil regardless of the loading configuration. This will be checked in the numerical example.

The use of the first law of thermodynamics applied to $\Omega$ also shows that the power dissipation is converted into heat. With the previous assumptions, the Helmholtz free energy, $\bar{\psi}$, can be written under the functional form:

$$
\bar{\psi}(\xi(\varepsilon), T)=\psi(\xi(\varepsilon))+\psi_{T}(T)
$$

in which $T$ is the temperature and $\xi(\varepsilon)$ denotes the function: $t \rightarrow \varepsilon(t)$. Then, the density of internal energy $(e)$ reads:

$$
e=\bar{\psi}+T s=\psi+e_{T}(T) \text { with } e_{T}=\psi_{T}-T \frac{d \psi_{T}}{d T}
$$

With these notations, the first law of thermodynamics for the whole structure reads: 


$$
\wp_{R R}^{s t r}+Q=\frac{d K}{d t}^{\text {medium }}+\int_{\Omega} \frac{d \psi}{d t} d \Omega+\int_{\Omega} \frac{d e_{T}}{d t} d \Omega
$$

with $Q$ characterizing the heat exchanges at the surface of the pavement. Since according to the previous developments $I=0$, substitution of Eq.(18) into Eq.(15) yields

$$
\int_{\Omega} \dot{\phi} d \Omega=\int_{\Omega} \frac{d e_{T}}{d t} d \Omega-Q
$$

Eq.(19) shows that the viscous dissipation is partly converted into heat and partly exchanged with the outside.

\subsection{Numerical calculation of the deflection and SRR}

To compute the SRR force from Eq.(7), the deflection for an asphalt pavement subjected to a moving load must be calculated. Prior to that, a constitutive law of the asphalt layers must be chosen, here taken as the Huet-Sayegh law.

\subsubsection{Huet-Sayegh thermo-viscoelastic model}

We use here the Huet-Sayegh (HS) model which was specifically developed to interpret the complex modulus tests performed on asphalt materials (Huet, 1963, 1999; Sayegh, 1965). It is represented by a purely elastic spring connected in parallel to two parabolic dampers (sensitive to temperature) in series with an elastic spring, as sketched in Fig. 3.

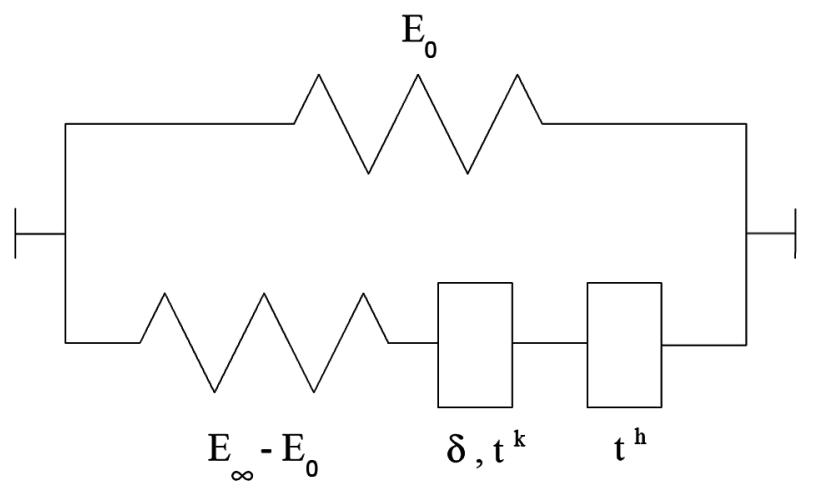

Figure 3. Schematic of the Huet-Sayegh rheological model

In the frequency domain, the complex modulus of the HS model reads:

$$
E^{*}=E_{0}+\frac{E_{\infty}-E_{0}}{1+\delta(i \omega \tau(\theta))^{-k}+(i \omega \tau(\theta))^{-h}}
$$


$E_{0}$ is the static elastic modulus, $E_{\infty}$ is the instantaneous elastic modulus, $k$ and $h$ are exponents of the parabolic dampers $(1>h>k>0)$, and $\delta$ is a positive non-dimensional coefficient. $\tau$ is a response time parameter which accounts for the equivalence principle between frequency $(\omega)$ and temperature $(\theta), \tau$ is governed by:

$$
\tau(\theta)=\exp \left(A_{0}+A_{1} \theta+A_{2} \theta^{2}\right)
$$

in which $A_{0}, A_{1}$ and $A_{2}$ are constant parameters.

The fitting of the HS parameters is done using the Viscoanalyse software (Chailleux et al., 2006). As an example, Fig. 4 shows the Black and the Cole-Cole diagrams relative to asphalt concretes AC 0-10 and AC 0-14 used in the applications of section 3. The values of the HS parameters corresponding to the plots of Fig. 4 are summarized in Table 2.
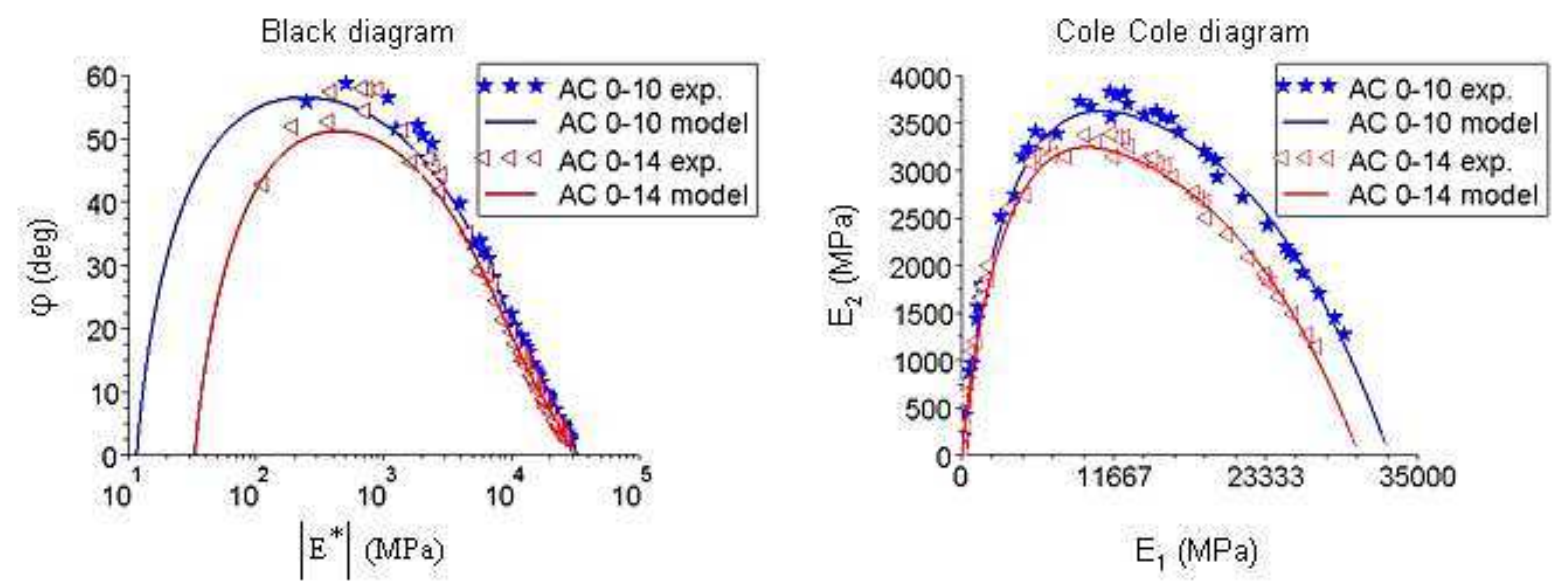

Figure 4. Black and Cole Cole diagrams for the two asphalt concretes denoted AC 0-10 and AC 0-14. The ViscoAnalyse software (Chailleux, 2006) is used to fit the Huet-Saygh model (straight lines) on the experimental data (symbols) stemming from complex modulus tests

Table 2. Values of the Huet-Sayegh parameters (LCPC ref. \#Aff450)

\begin{tabular}{llllllll}
\hline $\begin{array}{l}\text { E0 } \\
(\mathrm{MPa})\end{array}$ & $\begin{array}{l}\text { Einf } \\
(\mathrm{MPa})\end{array}$ & $\delta$ & $\mathrm{k}$ & $\mathrm{h}$ & $\begin{array}{l}\mathrm{A} 0 \\
(\mathrm{~s})\end{array}$ & $\begin{array}{l}\mathrm{A} 1 \\
\left(\mathrm{~s}^{\circ} \mathrm{C}^{-1}\right)\end{array}$ & $\begin{array}{l}\mathrm{A} 2 \\
\left(\mathrm{~s}^{\circ} \mathrm{C}^{-2}\right)\end{array}$ \\
\hline 11 & 32836 & 2.32 & 0.23 & 0.69 & 3.5059 & -0.3713 & 0.00157 \\
33 & 30501 & 2.23 & 0.21 & 0.66 & 3.9982 & -0.3747 & 0.00175 \\
\hline
\end{tabular}

\subsubsection{Calculation of the deflection using ViscoRoute@ 2.0}

The deflection in Eq.(7) is obtained using the ViscoRoute@ 2.0 software which has been described in previous works (e.g. Duhamel et al., 2005; Chabot et al., 2010; Chupin et al., 2010). Briefly, ViscoRoute $\subset 2.0$ is a numerical program dedicated to the computation of the 
response of a viscoelastic layered structure to moving loads. This program takes account of inertia forces and it allows to consider sliding (Chupin et al., 2010) or bonded conditions (Duhamel et al., 2005; Chabot et al., 2010) at the interface between layers. First in this program, a solution of the mechanical problem is sought in the wavenumber domain $\left(k_{1}, k_{2}\right)$ linked to the Fourier transforms in the $\mathrm{X}$ and $\mathrm{y}$ directions. The solution in the spatial domain is then obtained by means of Fast Fourier Transform (FFT) used to invert the solution calculated in the $\left(k_{1}, k_{2}\right)$ wavenumber domain. Note that, in some particular points, other adequate integration schemes are required to handle the integrable singularities that characterize the solution in the wavenumber domain (Chupin et al., 2010).

Using ViscoRoute $\bigcirc$ 2.0, numerical fields are computed at points of the rectangular grid utilized in the FFT computations. This mesh is not user-defined but automatically established during the running of the program in order to check convergence criteria of the built-in methods. Consequently, the grid points are generally not located on the load contour on which the integration in Eq.(7) must be performed. To interpolate the numerical fields (the deflection herein) on a load contour, we use the formula (in two dimensions) of the Shannon theorem, which reads at any point $(\mathrm{X}, \mathrm{y})$ of the load contour:

$$
\mathrm{w}^{*}(X, \mathrm{y}, \mathrm{z}) \approx \sum_{\mathrm{i}=-\infty}^{\infty} \sum_{\mathrm{j}=-\infty}^{\infty} \mathrm{w}^{*}(\mathrm{i} \Delta \mathrm{X}, \mathrm{j} \Delta \mathrm{y}, \mathrm{z}) \frac{\sin \frac{\pi}{\Delta \mathrm{x}}(\mathrm{X}-\mathrm{i} \Delta \mathrm{X})}{\frac{\pi}{\Delta \mathrm{x}}(\mathrm{X}-\mathrm{i} \Delta \mathrm{X})} \frac{\sin \frac{\pi}{\Delta \mathrm{y}}(\mathrm{y}-\mathrm{j} \Delta \mathrm{y})}{\frac{\pi}{\Delta \mathrm{y}}(\mathrm{y}-\mathrm{j} \Delta \mathrm{y})}
$$

where $\Delta X$ and $\Delta y$ are the spatial steps of the FFT in the $X$ and $y$ directions. The integral of Eq.(7) is calculated using a Gauss-Legendre quadrature rule on each segment $\left(X_{t}, y_{t}\right) \rightarrow\left(X_{t+1}, y_{t+1}\right)$.

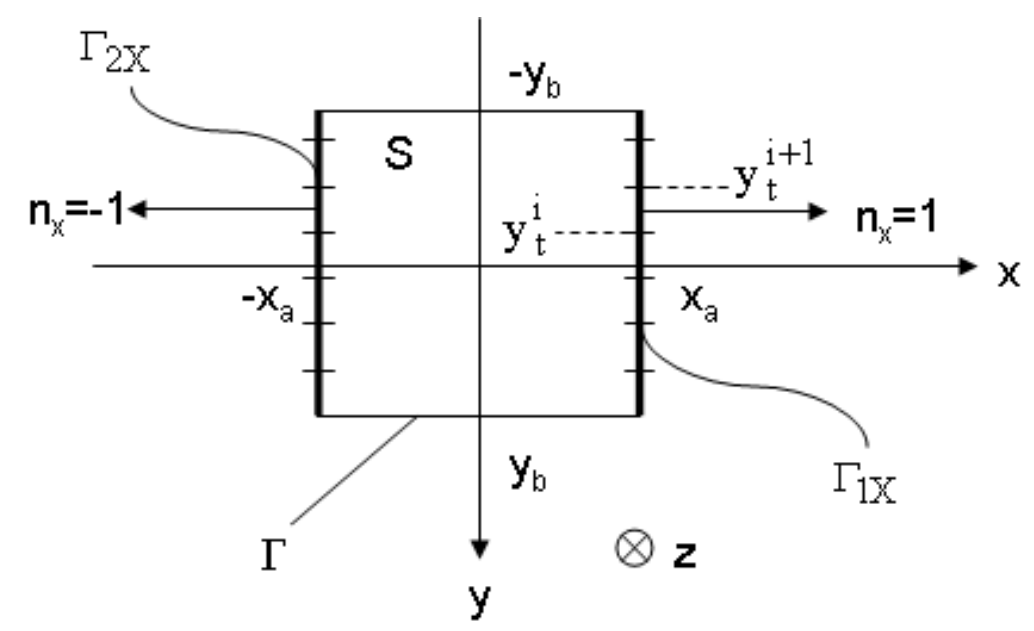


Figure 5. Discretization of a load imprint (the numerical integration for the determination of $\varsigma_{R R}^{s t r}$ is performed on $\left.\Gamma_{1 x} \cup \Gamma_{2 x}\right)$

As illustrated in Fig. 5, only rectangular-shaped loads are considered in this article. Then, only contours parallel to the $\mathrm{y}$-axis (located in $\pm X_{a}$ in Fig. 5 and for which $n_{x}= \pm 1$ ) are considered and discretized in $n$ segments. A 2-point rule is applied on each of them. Then, the SRR force is obtained from discrete values of the deflection as follows:

$$
\mathrm{F}_{\mathrm{RR}}^{\mathrm{str}}=\mathrm{p} \sum_{\mathrm{t}=1}^{\mathrm{n}} \frac{\left|\mathrm{y}_{\mathrm{t}+1}-\mathrm{y}_{\mathrm{t}}\right|}{2} \sum_{j=1}^{2} \omega_{j}\left[\begin{array}{l}
\mathrm{w}^{*}\left(-\mathrm{X}_{\mathrm{a}}, \frac{\mathrm{y}_{\mathrm{t}+1}-\mathrm{y}_{\mathrm{t}}}{2} \mathrm{p}_{\mathrm{j}}+\frac{\mathrm{y}_{\mathrm{t}+1}+\mathrm{y}_{\mathrm{t}}}{2}, 0\right) \\
-\mathrm{w}^{*}\left(\mathrm{X}_{\mathrm{a}}, \frac{\mathrm{y}_{\mathrm{t}+1}-\mathrm{y}_{\mathrm{t}}}{2} \mathrm{p}_{\mathrm{j}}+\frac{\mathrm{y}_{\mathrm{t}+1}+\mathrm{y}_{\mathrm{t}}}{2}, 0\right)
\end{array}\right]
$$

in which $\omega_{j}$ and $p_{j}$ denote the weights and the abscissas of the quadrature rule.

In the case of multiple loads, ViscoRoute $\subset 2.0$ computations are performed considering all the loads (with cumulative effects for the deflection) and the Shannon theorem is applied to each of the load contours parallel to the y-axis. $F_{R R}^{s t r}$ is then obtained using Eq.(8). For several independent groups of interacting loads, Eq.(9) is further used to get the global $F_{R R}^{s t r}$. An automatic procedure has been developed for the computation of $F_{R R}^{s t r}$ for any set of loads.

\section{Validation of the method and application}

The previous developments were first validated in the case of a homogeneous half-space before being applied to the case of a typical thick asphalt pavement, as used for heavy traffic roads.

\subsection{Computation of the SRR force in the case of a homogeneous half-space}

The case of a homogeneous half-space is used to validate the computation procedure of $F_{R R}^{s t r}$ and to illustrate the fact that the power dissipation within an elastic medium is equal to zero whatever the loading configuration. We also show in this section that accounting for kinetic energy in Eq.(7) or (15) barely changes the magnitude of $F_{R R}^{s t r}$.

\subsubsection{Zero dissipation property of an elastic pavement}

Here, we aim at illustrating the fact that the SRR force within an elastic medium is null for any loading configuration, as shown in section 2.2 when inertia forces are neglected. This result is 
evident for symmetric loading since in that case the deflection is also symmetric and, hence, the integral in Eq.(7) is zero. However, this property is not such obvious for any given load.

Consequently, this was checked on the example of Fig. 6(b) in the case of a homogeneous half-space $(\mathrm{E}=9000 \mathrm{MPa}$ and $\mathrm{v}=0.35)$.

For each load, the deflection computed at rear and front is plotted in Fig. 7 with the corresponding value of $F_{R R}^{s t r}$ obtained from the algebraic area enclosed between the two curves. The total structure-induced power dissipation obtained by summation is found equal to $-0.007 \mathrm{~W}$, which can be considered as zero compared to the contribution of each term. This corroborates, within the limits of numerical accuracy, the zero-dissipation property of elastic media.

(a)

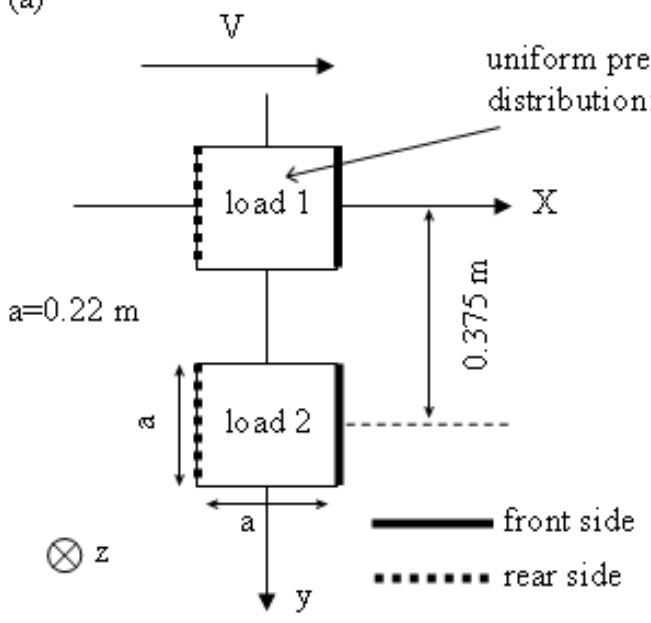

total weigth: $65 \mathrm{kN}$ (b)

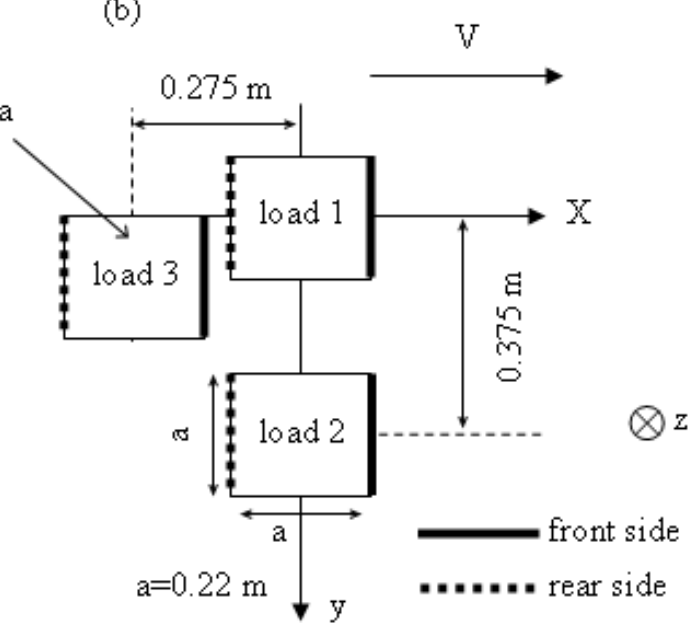

total weigth: $94.5 \mathrm{kN}$

Figure 6. Top view of the loading configurations: (a) configuration corresponding to dual tires of a half-axle (the French standard for truck: $65 \mathrm{kN}$ ), (b) non symmetric loading configuration used to illustrate the zero-dissipation property of an elastic medium 

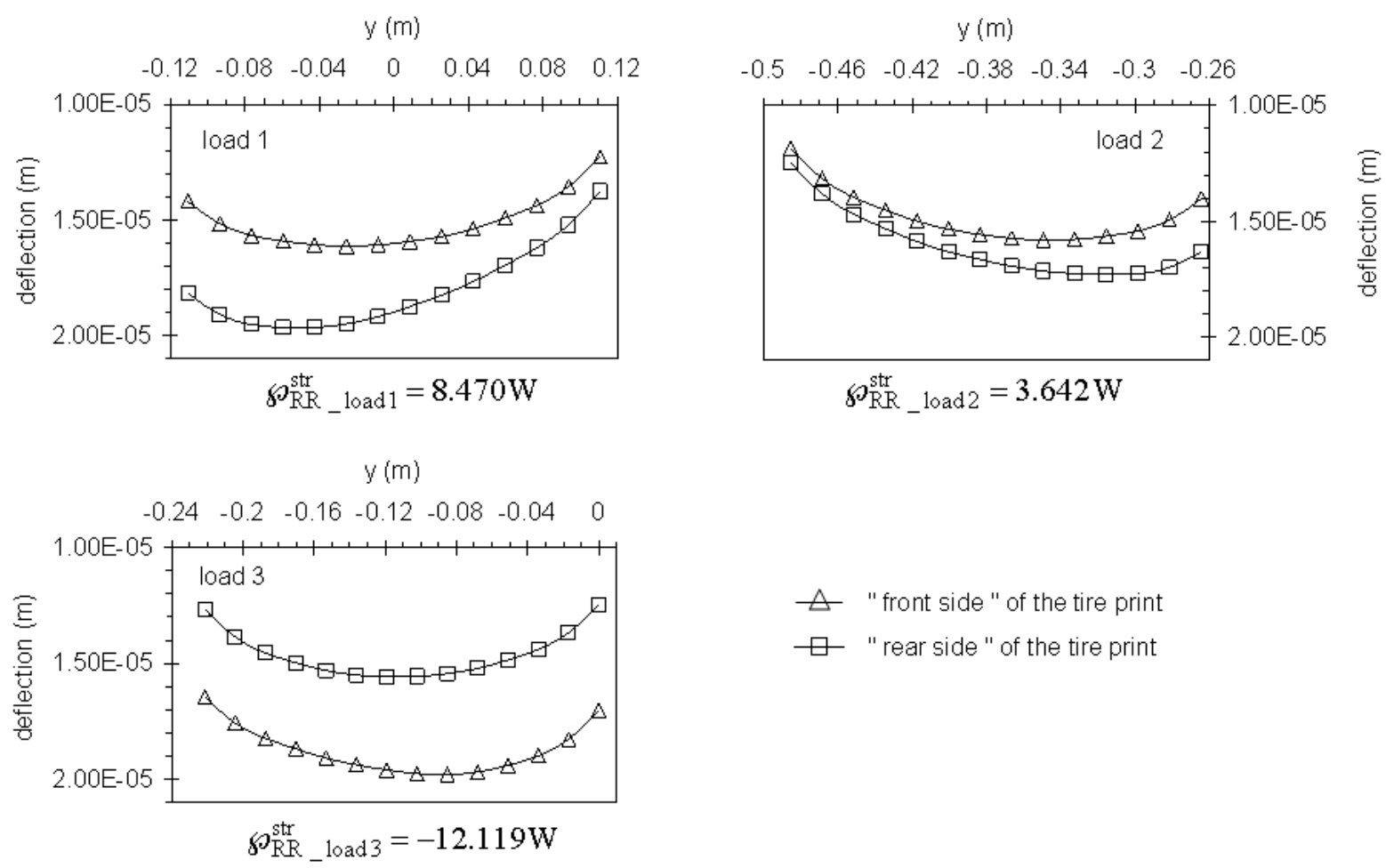

Figure 7. Deflection at the front and the rear sides of each individual load of the unsymmetrical loading configuration (elastic semi-infinite half-space)

\subsubsection{Validation for a Huet-Sayegh viscoelastic half space}

When ViscoRoute@ 2.0 was programmed an analytical solution was developed in parallel to validate the implementation in the simple case of a homogeneous viscoelastic half-space (Chabot and Piau, 2001). Then, we also used this solution to compute $F_{R R}^{s t r}$ and to validate the general method based on ViscoRoute $\bigcirc 2.0$ calculations. The parameter values used in the comparison shown here are those provided in Table 2 (AC 0-14) except for $E_{0}$ which is set equal to zero as required by the analytical solution. The moving loads applied at the surface of the viscoelastic medium are representative of a half-axle of a standard truck (Fig. 6(a)). The load speed is set to $20 \mathrm{~m} / \mathrm{s}$ and the computations are performed for a temperature of $15^{\circ} \mathrm{C}$. The deflection profiles in the $y$-direction for $X=0.11 \mathrm{~m}$ and $\mathrm{X}=-0.11 \mathrm{~m}$ obtained with both methods are very close to each other and plotted in Fig. 8. The $F_{R R}^{s t r}$ values, proportional to the hatched areas, are equal to $0.60 \mathrm{~N}$ and $0.65 \mathrm{~N}$ for the methods based on ViscoRoute $\odot 2.0$ and the analytical solution, respectively. The slight difference is due to the difference in deflection noticed between the two methods and arising from the finite spatial step of the FFT used in ViscoRoute $\bigodot 2.0$. 


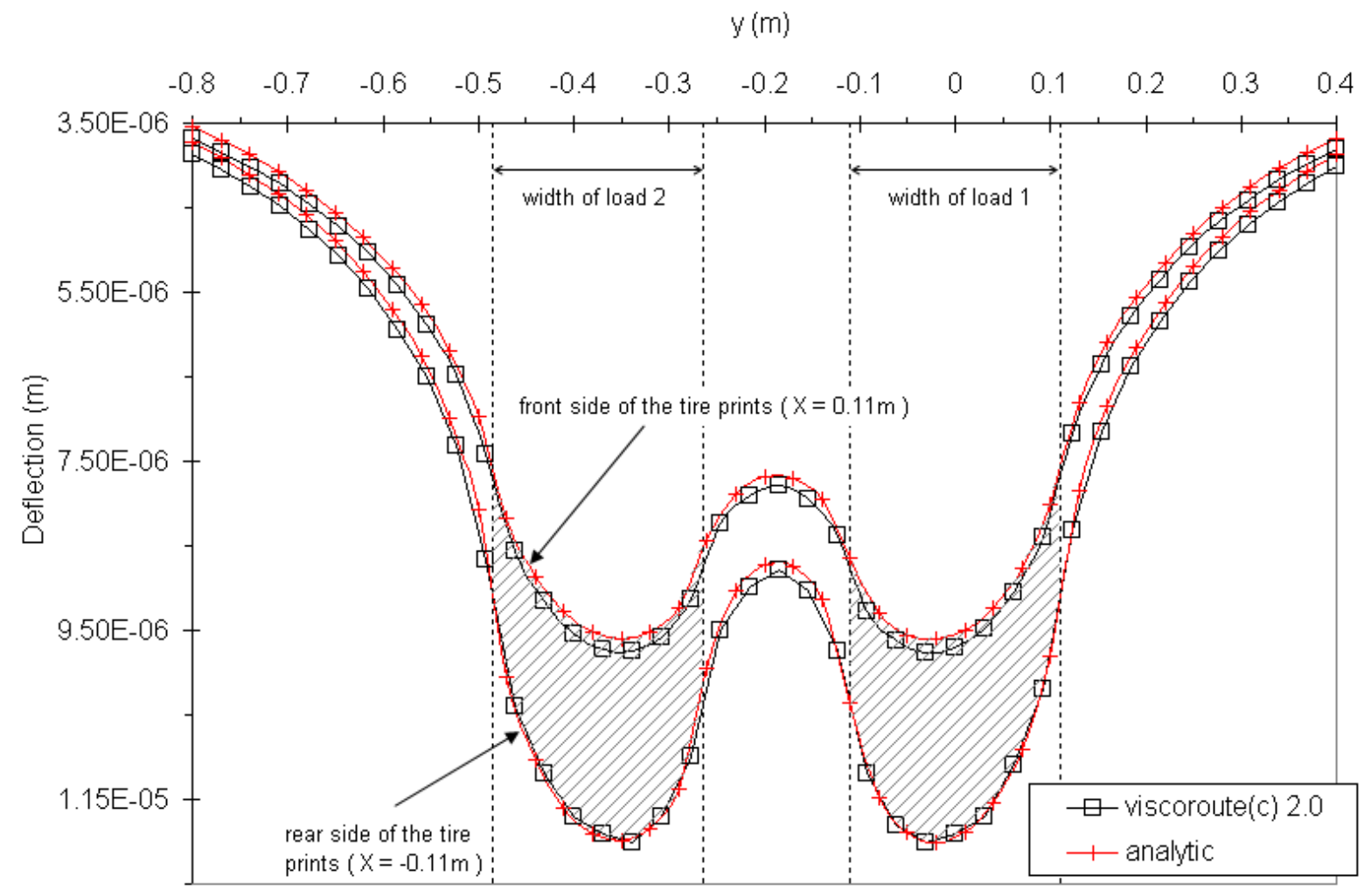

Figure 8. Deflection of the viscoelastic half-space at the front and the rear sides of the tire prints shown in Figure 6(a): comparison between ViscoRoute $(2)$ and the analytical solution $\left(\mathrm{V}=20 \mathrm{~m} / \mathrm{s}, \theta=15^{\circ} \mathrm{C}\right)$

As a remark, if one computes for this example $F_{R R}^{s t r}$ for a single wheel and then multiplies the computed value by two, the total $F_{R R}^{s t r}$ is found equal to $0.59 \mathrm{~N}$ (using the analytical method) which is close to the $F_{R R}^{s t r}$ computed for dual wheels. This indicates that in the present case the two loads almost do not interact between themselves in terms of SRR.

\subsection{Computation of $F_{R R}^{s t r}$ in the case of a representative flexible pavement}

In this section, the SRR is computed for a typical thick asphalt pavement (SETRA-LCPC, 1997; Corte and Goux, 1996) composed of three layers defined as follows: a surface course made up of a semi-coarse asphalt concrete (AC 0-10), a base layer of asphalt concrete (AC $0-14$ ) and a pavement foundation. The two first layers have a viscoelastic behavior and the pavement foundation is assumed to follow Hooke's law. The pavement structure is depicted in Fig. 9 and the values of the viscoelastic parameters are those of Table 2. The moving load applied on this pavement is the half-axle of Fig. 6(a). However, the figures below are 
extrapolated to a 400-kN truck $\left(\mathrm{F}_{\mathrm{RR} \_400}^{\text {str }}\right)$ using Eq.(9). The influence of temperature and speed on $\mathrm{F}_{\mathrm{RR} \_400}^{\mathrm{str}}\left(\right.$ or $\left.\wp_{\mathrm{RR}_{-} 400}^{\mathrm{str}}\right)$ is specially discussed.

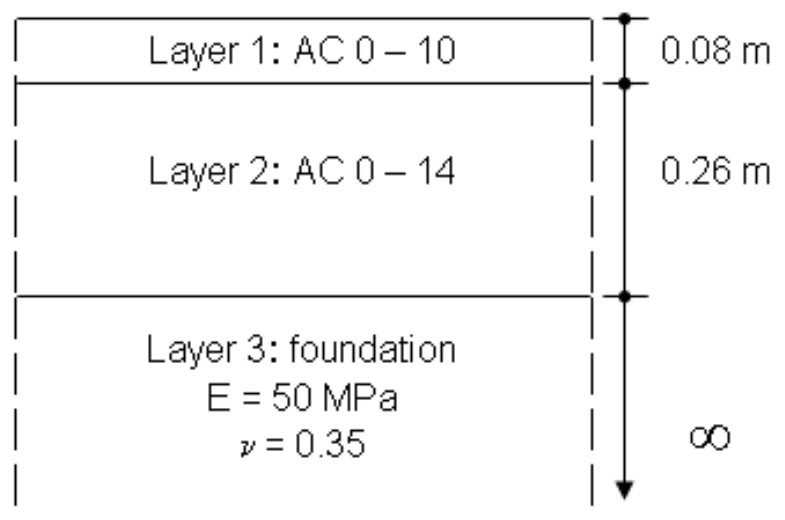

Figure 9. Schematic representation of the thick asphalt pavement

\subsubsection{Calculation of SRR as a function of the pavement temperature}

The evolution of $\mathrm{F}_{\mathrm{RR}_{-} 400}^{\mathrm{str}}$ with the pavement temperature (assumed uniform) is shown in Fig. 10 , for a truck speed of $20 \mathrm{~m} / \mathrm{s}$. As expected, the evolution of $\mathrm{F}_{\mathrm{RR}_{-} 400}^{\mathrm{str}}\left(\right.$ resp. $\left.\wp_{\mathrm{RR} \_}^{\mathrm{str}} 400\right)$ is found to increase strongly with the pavement temperature since the stiffness of asphalt materials decreases with temperature, leading to higher deflections. However, it can be observed that even at $40^{\circ} \mathrm{C}, \mathrm{F}_{\mathrm{RR}_{-} 400}^{\mathrm{str}}$ is less than $200 \mathrm{~N}$ and $\wp_{\mathrm{RR}_{-} 400}^{\mathrm{str}}$ is lower than $4 \mathrm{~kW}$. Let us compare this last figure with the one given in the literature that estimates the global rolling resistance to $12 \%$ of the energy available in the fuel of a truck $(20 \mathrm{~m} / \mathrm{s})$, i.e. approximately $100 \mathrm{~kW}$ at maximum power output (Table 1). It shows that the contribution of the structure to the total rolling resistance is less than 5\%. By the way, it can be deduced that other parameters affecting the rolling resistance (tire dissipation, texture...) account for the $95 \%$ left. Still, $\wp_{\mathrm{RR}_{-} 400}^{\mathrm{str}}$ is less than $1.5 \%$ of the engine power of the truck $(335 \mathrm{~kW})$ or $0.5 \%$ of the theoretical energy available in the fuel. In similar conditions ( $V=20 \mathrm{~m} / \mathrm{s}$ and summer conditions) Zaabar and Chatti (2011) measured values for the extra fuel consumption that were not statistically significant, confirming the smallness of this quantity in this case. This also explains the difficulty of measuring the sole effect of the structure given its small contribution to the rolling resistance. 


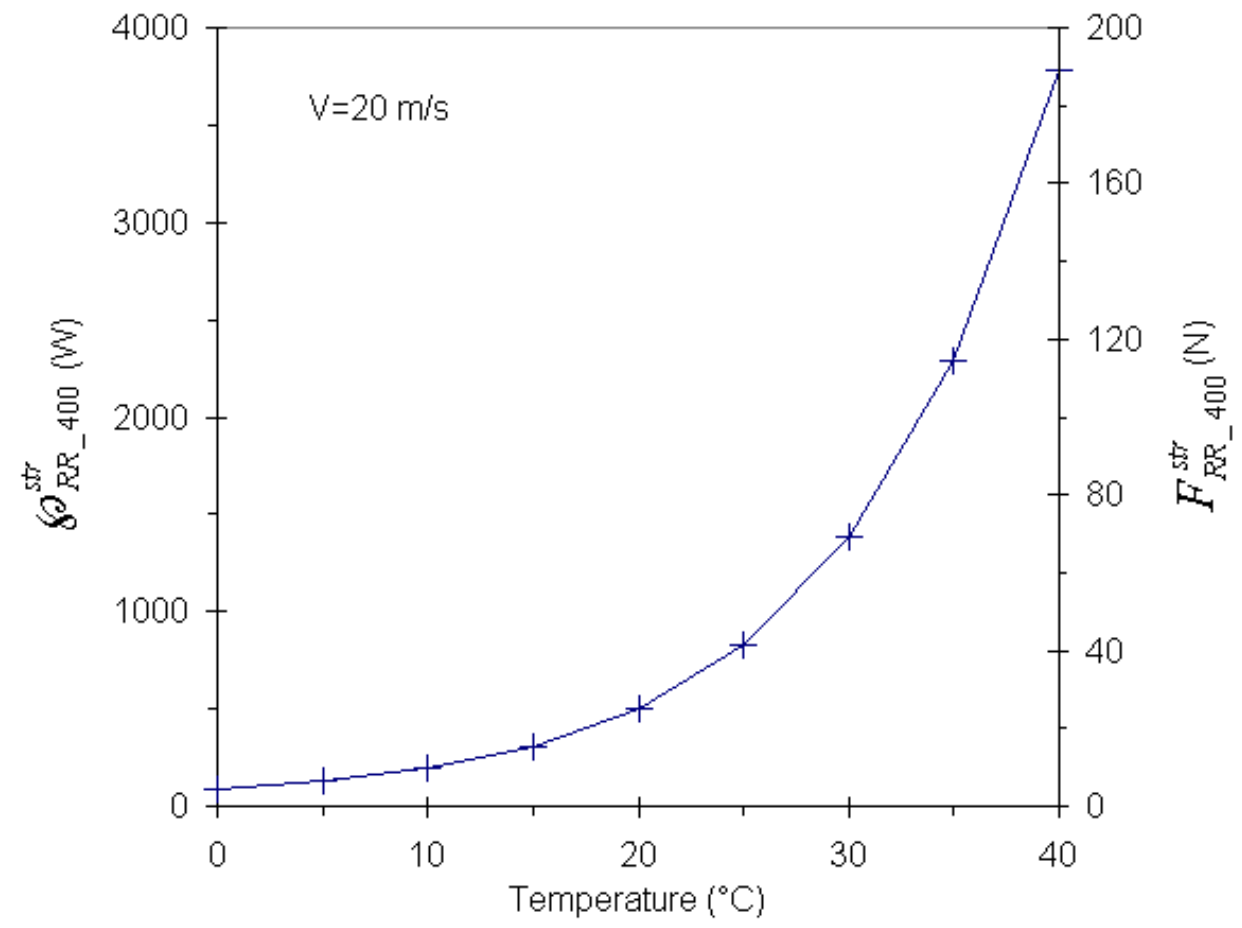

Figure 10. Structure-induced power dissipation $\left(\wp_{R R_{-} 400}^{s t r}\right)$ and SRR force $\left(F_{R R_{-} 400}^{s t r}\right)$ computed for a thick asphalt pavement as a function of temperature $(\mathrm{V}=20 \mathrm{~m} / \mathrm{s})$

Notes:

(i) For the present structure, the power dissipation at $15^{\circ} \mathrm{C}$ for one half-axle is found equal to $50 \mathrm{~W}$ which is close to 4 times the power dissipation computed for the homogeneous half-space under the same load; the material properties of the homogeneous medium being the same as those of layer 2 (AC 0-14) of the flexible pavement. It shows that the case of a homogeneous half-space should not be systematically considered as the upper bound for the estimation of the structure-induced power dissipation. This is due to the fact that the realistic pavement model because of its foundation is less stiff than the homogeneous half-space. More generally, for a two-layer structure (AC 0-14 base layer + elastic subgrade) the SRR dependence on the thickness of the asphalt layer is shown in Fig. $11\left(\mathrm{~V}=20 \mathrm{~m} / \mathrm{s}, \mathrm{T}=40^{\circ} \mathrm{C}\right)$. A peak is observed for a thickness around $5 \mathrm{~cm}$. However such a structure would not be convenient for heavy traffic roads and thus not used in practice.

(ii) In section 2, we assumed that the horizontal surface forces could be neglected in the computation of the pavement response. This assumption can now be justified. Indeed, combining Eq.(1) and (2) makes it possible to derive the equality $\tau=\wp_{R R}^{s t r} / S V$ for a towed 
wheel and the inequality $\tau<\wp_{R R}^{s t r} / S V$ for a driving wheel. In both cases, the ratio of the shear stress to the vertical pressure verifies: $\tau / p<0.05 \%$.

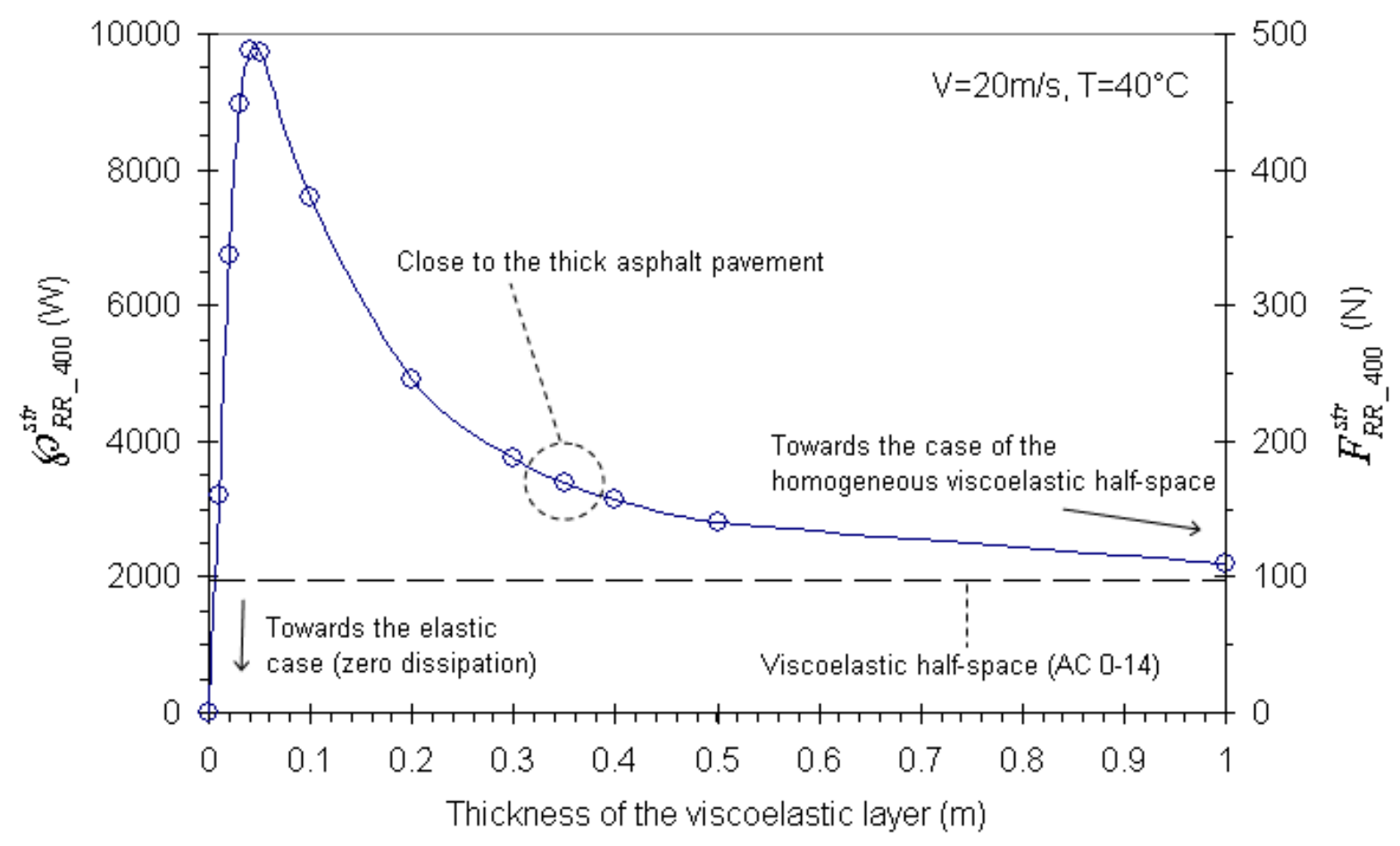

Figure 11. SRR versus thickness of the viscoelastic layer of a two-layer structure composed of an elastic $(\mathrm{E}=50 \mathrm{MPa})$ and a viscoelastic layer (AC 0-14). SRR is computed for $\mathrm{V}=20 \mathrm{~m} / \mathrm{s}$ and $\mathrm{T}=40^{\circ} \mathrm{C}$

\subsubsection{Influence of speed}

As when temperature increases, the viscous behavior of asphalt layers becomes more pronounced as the speed of loads applied on the pavement structure decreases. This implies that low speeds produce higher $\mathrm{F}_{\mathrm{RR}}^{\mathrm{str}}$ and therefore, from this standpoint, require more energy for a given travel distance. The evolution of $\mathrm{F}_{\mathrm{RR} \_}^{\mathrm{str}} 400$ with speed is plotted in Fig. 12 for a temperature of $40^{\circ} \mathrm{C}$. A ratio of about 2.5 is found between $\mathrm{F}_{\mathrm{RR}_{-} 400}^{\mathrm{str}}$ computed at $35 \mathrm{~m} / \mathrm{s}$ $(\sim 150 \mathrm{~N})$ and $5 \mathrm{~m} / \mathrm{s}(\sim 400 \mathrm{~N})$. However, even under these unfavorable condition (low speed and high pavement temperature), the SRR remains less than $10 \%$ of the global rolling resistance. The influence of speed on $\wp_{\mathrm{RR}_{-} 400}^{\mathrm{str}}$ is also shown in Fig. 12. Note that due to the factor $1 / V$ between $\wp_{\mathrm{RR}_{-} 400}^{\mathrm{str}}$ and $\mathrm{F}_{\mathrm{RR}_{-} 400}^{\mathrm{str}}$, the graph this time is found to increase with speed, but as mentioned previously $\wp_{\mathrm{RR}_{-} 400}^{\mathrm{str}}$ is less representative of the SRR than $\mathrm{F}_{\mathrm{RR}_{-}}^{\mathrm{str}} 400$. 


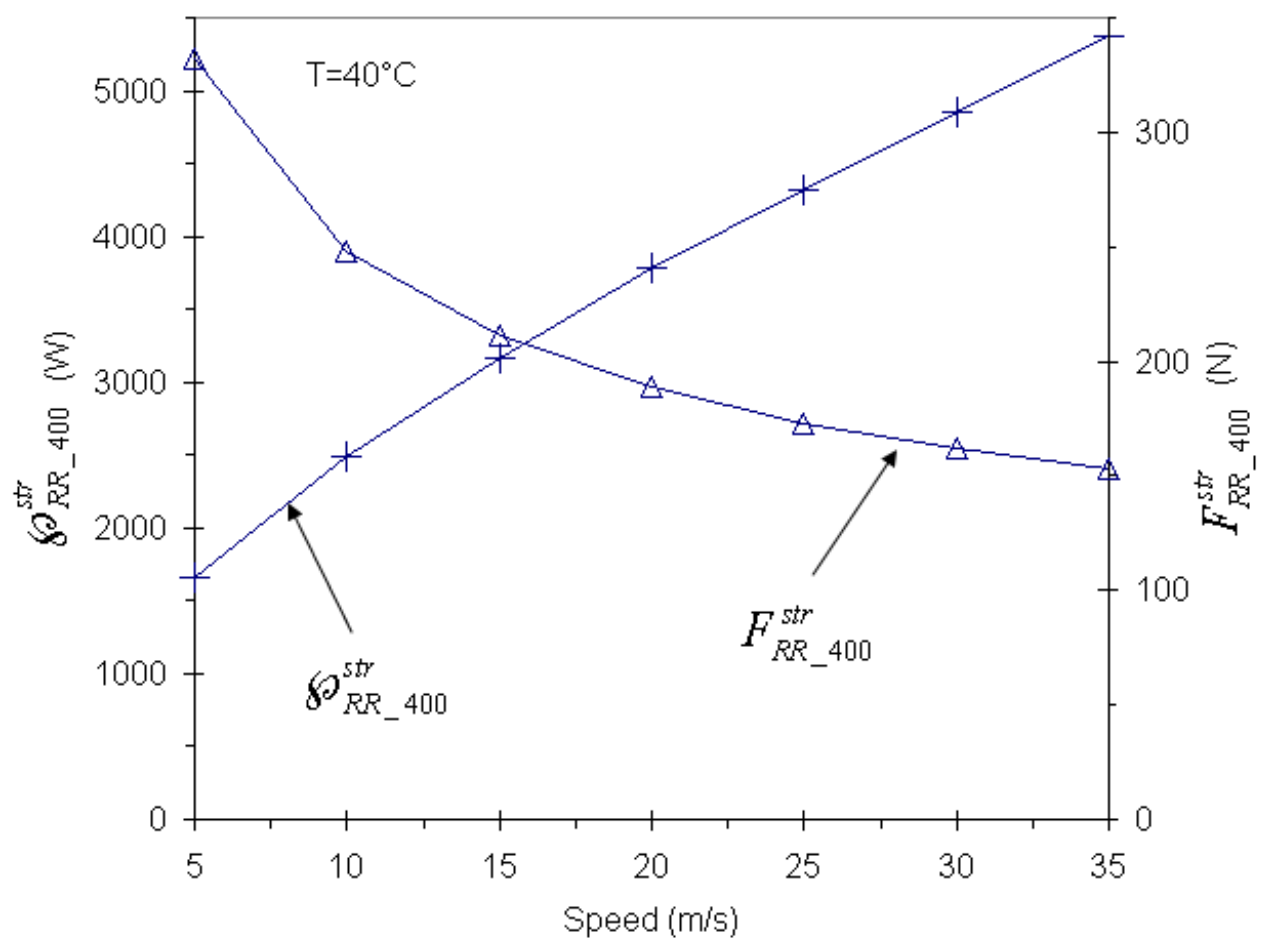

Figure 12. Evolution of the SRR force $\left(F_{R R_{-} 400}^{s t r}\right)$ and the structure-induced power dissipation $\left(\wp_{R_{-} 400}^{s t r}\right)$ computed for a thick asphalt pavement as a function of speed $\left(\theta=40^{\circ} \mathrm{C}\right)$

\section{Extrapolation of the discussion using a non-dimensional analysis}

A non-dimensional analysis of SRR for a homogeneous viscoelastic medium makes it possible to summarize the influence of the main factors on SRR and also to extrapolate the previous calculations to the case of passenger vehicles.

The displacement field in such a medium solicited by a single moving load is a function of several quantities that, according to the model assumptions, can be listed as follows: the uniform contact pressure $(p)$ resulting from the applied load, the characteristic length $(a)$ and the velocity $(V)$ of the applied load, the complex modulus of the linear viscoelastic model (characterized by $E_{\infty}$ for the Huet-Sayegh model with $E_{0}=0$, and fixed values of $\delta, k, h$ ), and the retardation time parameter $(\tau(\theta))$ that depends, in the present article, on temperature. This leads to the following expression of the deflection:

$$
w^{*}(x, y, z=0)=a \frac{p}{E_{\infty}} \cdot f\left(\frac{V \tau(\theta)}{a}, \frac{x}{a}, \frac{y}{a}\right)
$$


with $f$ being a non-dimensional function. Using Eq.(24) to substitute in Eq.(7) yields:

$$
F_{R R}^{s t r}=-\frac{a p^{2}}{E_{\infty}} \int_{\Gamma} f\left(\frac{V \tau(\theta)}{a}, \frac{x}{a}, \frac{y}{a}\right) \cdot n_{x} d l=\frac{a^{2} p^{2}}{E_{\infty}} g\left(\frac{V \tau(\theta)}{a}\right)
$$

in which $g(V \tau(\theta) / a)$ is a non-dimensional function. Furthermore, it is convenient in the present analysis to consider the weight of the applied load (denoted W) to replace in Eq.(25) that becomes (without changing the notation of function $g$ which is proportional to the one above):

$$
F_{R R}^{s t r}=\frac{p W}{E_{\infty}} g\left(\frac{V \tau(\theta)}{a}\right)
$$

This function $(g)$ is a priori unknown but can be determined numerically by computing the SRR force as a function of $V \tau(\theta) / a$, for any given particular case. This was done using $\mathrm{a}=0.22 \mathrm{~m}$ (square-shaped load), $\mathrm{W}=32500 \mathrm{~N}, \mathrm{~V}=20 \mathrm{~m} / \mathrm{s}$ and by varying the temperature (Fig. 13).

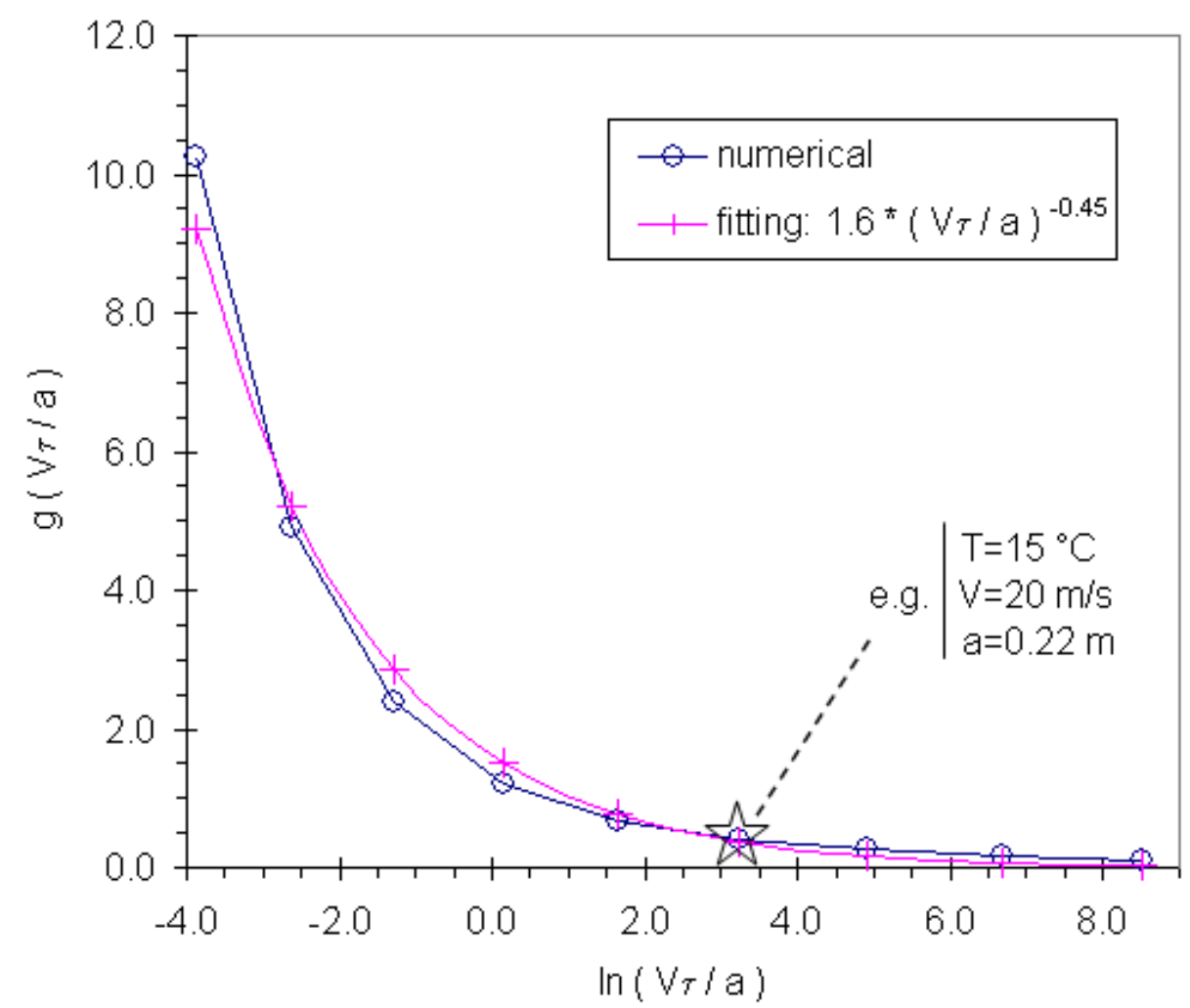

Figure 13. Evolution of the non-dimensional function $g$ as a function of the non-dimensional parameter $V \tau / a$ (one wheel on a homogeneous viscoelastic medium) 
For convenience, this function is fitted by the following power-law (cross symbols in Fig. 13):

$$
g\left(\frac{V \tau}{a}\right) \approx 1.6\left(\frac{V \tau}{a}\right)^{-\alpha}
$$

In the present case $\alpha=0.45$. It can be checked that the shape and the values of $\mathrm{g}$ corroborate the previous discussions about the effect of temperature and speed on SRR. But it also allows the comparison of the SRR force for a heavy truck $\left(F_{R R_{-} H T}^{s t r}\right)$ with that of a passenger car ( $F_{R R_{-} C}^{s t r}$ ) whose weight is supposed to be $10 \mathrm{kN}$. The ratio $R_{H T / C}$ between the SRR forces of such vehicles reads:

$$
\mathrm{R}_{\mathrm{HT} / \mathrm{C}}=\frac{\mathrm{F}_{\mathrm{RR} \_\mathrm{HT}}^{\mathrm{str}}}{\mathrm{F}_{\mathrm{RR} \_\mathrm{C}}^{\mathrm{str}}}=\frac{\mathrm{W}_{\mathrm{HT}}}{\mathrm{W}_{\mathrm{C}}} \frac{\mathrm{p}_{\mathrm{HT}}}{\mathrm{p}_{\mathrm{C}}}\left(\frac{\mathrm{a}_{\mathrm{HT}}}{\mathrm{a}_{\mathrm{C}}}\right)^{\alpha}
$$

in which subscripts C and HT stand for car and heavy truck, respectively. $R_{H T / C}$ is evaluated using the parameter values given in Table 3 taken as estimates of the real values. These figures yield $R_{H T / C} \approx 220$, indicating that the SRR force for a passenger car is approximately $1 / 220$ times that of a heavy truck. In particular, for $\mathrm{V}=20 \mathrm{~m} / \mathrm{s}$ and $\mathrm{T}=40^{\circ} \mathrm{C}, F_{R R_{-} C}^{s t r}$ can be estimated to $0.86 \mathrm{~N}$. Besides, considering that heavy trucks represent roughly $15 \%$ of the road traffic in Europe (Perriot, 2008), the SRR ratio between cars and trucks is roughly 1/30.

Table 3. Weight (W), contact pressure ( $\mathrm{p}$ taken equal to the tire inflation pressure) and characteristic dimension (a) used in the non-dimensional analysis

\begin{tabular}{lccc}
\cline { 2 - 4 } & $\mathrm{W}(\mathrm{kN})$ & $\mathrm{p}(\mathrm{MPa})$ & $\mathrm{a}(\mathrm{m})$ \\
\hline Car & 10 & 0.2 & 0.11 \\
\hline Heavy truck & 400 & 0.8 & 0.22 \\
\hline
\end{tabular}

\section{Conclusion}

In this article, we have presented a method to derive the SRR for a vehicle driving along a flat (non-damaged) asphalt pavement. We recall that the SRR (or Structure-induced Rolling Resistance) evaluates the contribution of the structure to the global rolling resistance and is obtained by neglecting all effects other than the viscous dissipation of the bituminous layers induced by the pavement deflection under traffic loads. It is shown that SRR is proportional to the integral over contours of the applied loads of the deflection times the outward normal to 
these contours. The application of the developed method to the study of a typical thick asphalt pavement leads to the following conclusions:

(1) As expected, the SRR of asphalt pavements increases with temperature (exponential-like) and decreases with speed.

(2) However, even under unfavorable conditions, i.e. for a uniform temperature of $40^{\circ} \mathrm{C}$ within the pavement, the SRR for a 40 -ton truck driving at $20 \mathrm{~m} / \mathrm{s}$ is less than $0.5 \%$ of the total energy available in the fuel. Now, the combination of the results of Figures 10, 11 and 12 shows that a value of about $1 \%$ or $2 \%$ might be reached theoretically for a small thickness of the base course, a slow driving speed and high temperature conditions. However, the condition about thickness is not applicable to the case of high-traffic roads.

(3) The small contribution of the pavement structure to the fuel consumption explains the difficulty of measuring the part of the pavement structure in the rolling resistance.

(4) A priori, the substantial difference in rolling resistance between asphalt and rigid pavements reported in some studies can not be explained by a structural effect (at least for soils considered as non-dissipative).

(5) A non-dimensional analysis shows that the part of SRR in the energy consumption of road traffic is about 30 times less for passenger cars than for trucks.

\section{References}

Benbow E, Iaquinta J, Lodge R, Wright A (2007) Investigation of the effects of pavement stifnness on fuel consumption, published project report ppr253, TRL Limited

Bendtsen H (2004) Rolling resistance, fuel consumption and emissions: a literature review, SILVIA project report

Chabot A, Chupin O, Deloffre L, Duhamel D (2010) Viscoroute 2.0: a tool for the simulation of moving load effects on asphalt pavement. RMPD Special Issue on Recent Advances in Numerical Simulation of Pavements 11(2):227-250

Chabot A, Piau JM (2001) Calcul semi-analytique d'un massif viscoélastique soumis à une charge roulante rectangulaire, 1ere conférence international Albert Caquot, Paris, France

Chailleux E, Ramond G, Such C, de la Roche C (2006) A mathematical-based master-curve construction method applied to complex modulus of bituminous materials. Roads Materials and Pavement Design 7 (EATA Special Issue):75-92 
Chupin O, Chabot A, Piau JM, Duhamel D (2010) Influence of sliding interfaces on the response of a layered viscoelastic medium under a moving load. International Journal of Solids and Structures, 47:3435-3446

Corte JF, Goux MT (1996) Design of pavement structures: the French technical guide. Transport Research Report, 1539:116-124

Duhamel D, Chabot A, Tamagny P, Harfouche L (2005) ViscoRoute: visco-elastic modeling for asphalt pavements. Bulletin des Laboratoires des Ponts et Chaussées (http://www.lcpc.fr/en/sources/blpc/index.php), 258-259:89-103

EAPA (2004) Environmental impacts and fuel efficiency of road pavements, EAPA \& Eurobitume Industry Report, March 2004

Glaeser KP (2005) Measurements of rolling resistance of tyres on road surfaces. IEA workshop, Paris, November $15-16$

Hammoum F, Chabot A, St-Laurent D, Chollet H, Vulturescu B (2010) Effects of accelerating and decelerating tramway loads on bituminous pavement. Materials and Structures, 43:1257-1269

Huet C (1963) Etude par une méthode d'impédance du comportement viscoélastique des matériaux hydrocarbonés. Université de Paris (France), Ph.D. thesis

Huet C (1999) Coupled size and boundary-condition effects in viscoelastic heterogeneous and composite bodies. Mechanics of Materials, 31:787-829

Igwe EA, Ayotamuno MJ, Okparanma RN, Ogaji SOT, Probert SD (2009) Road-surface properties affecting rates of energy dissipation from vehicles. Applied Energy, 86:1692-1696

Lemaitre J, Chaboche JL (1996) Mécanique des matériaux solides. Ed. Dunod, Paris

Lepert P, Brillet F (2009) The overall effects of road works on global warming gas emissions. Transportation Research Part D, 14: 576-584

Perriot A (2008) Route et énergie: comment la chaussée peut peser sur la consummation des véhicules. Colas report.

Sandberg T (2001) Heavy truck modelling for fuel consumption. Simulations and Measurements. Thesis No. 924, Linköping University, Sweden 
Sayegh G (1965) Contribution à l'étude des propriétés viscoélastiques des bitumes purs et des bétons bitumineux. Faculté des Sciences de Paris (France), Ph.D. thesis

SETRA-LCPC (1997). French Design Manual For Pavement Structures, Ed. Laboratoire Central des Ponts et Chaussées and Service d'Etudes Techniques des Routes et Autoroutes

Staverman AJ, Schwarzl P (1952a). Thermodynamics of viscoelastic behaviour (model theory), Proceedings of Academy of Science, The Netherlands, 55: 474-485

Staverman AJ, Schwarzl P (1952b) Non-equilibrium thermodynamics of visco-elastic behaviour, Proceedings of Academy of Science, The Netherlands, 55: 486-492

Taylor GW, Patten JD (2006) Effects of pavement structure on vehicle fuel consumption - phase III, NRC-CNRC technical report

Zaabar I, Chatti K (2010) Calibration of HDM-4 Models for Estimating the Effect of Pavement Roughness on Fuel Consumption for U. S. Conditions. Transportation Research Record: Journal of the Transportation Research Board, Issue 2155:105-116

Zaabar I, Chatti K (2011) A field investigation of the effect of pavement surface conditions on fuel consumption, Proceedings of the 90th Annual Meeting of Transportation Research Board, Washington D.C.

Zaniewski J (1989) Effect of pavement surface type on fuel consumption. Portland Cement Association 